\title{
Control of Redox Homeostasis in Tick Blood Feeding*
}

\author{
Gabriela Alves Sabadin ', Marina Amaral Xavier' \& Itabajara da Silva Vaz Jr. ${ }^{1,2,3}$
}

\begin{abstract}
Introduction: Ticks are hematophagous ectoparasites that cause significate economical losses. Acaricide application is the main method to tick control. However, it causes environmental contamination and selects resistant ticks. The immunological control represents a suitable method to replace or complement acaricide application. During its life cycle, female ticks ingest large amounts of blood, which contains toxic components able to damage biomolecules. Understanding which molecular mechanisms and proteins are involved in avoid damages caused by blood intake in ticks and other hematophagous arthropods could help to found potential candidate antigens to compose an anti-tick vaccine.

Review: Hemoglobin comprises almost $20 \%$ of mammalian blood proteins, its hydrolysis during tick digestion increases total free heme that can potentially generate reactive oxygen species (ROS), which easily oxidize lipids, proteins and DNA, modifying they structure and function. Lipids are more susceptible to high ROS levels. It can lead to membrane instability and cell death. Protein modifications caused by ROS can promote the protein loss of function and cell disturbance, however, it can also work as post-translational modifications, acting in cell signaling processes. DNA presents more efficient protective mechanisms against ROS, but damages can lead to double helix rupture. Oxidative stress is defined as a disturbance in the balance between the production and elimination of ROS, in favor of ROS production, leading to a disruption in redox homeostasis and/or molecular damage. Despite the well-recognized heme oxidative power and its already demonstrated cytotoxicity, ticks are able to feed on blood, controlling the redox homeostasis without causing oxidative stress. This occurs because ticks developed physiological adaptations to transport, store, metabolize and secrete toxic components from the diet. The strategies, such as heme compartmentalization in specialized organelles, and heme and iron carrying proteins are shared with most of other hematophagous. Interesting, heme degradation, a mechanism commonly described in hematophagous arthropods, showed to be absent in ticks. Moreover, there is a lack of key enzymes from the heme degradation pathway in tick genomes. As expected, antioxidant enzymes are often involved in homeostasis redox control. They act in a convenient way, eliminating physiological and non-physiological produced ROS. Despite their essential role in nonhematophagous organisms, antioxidant enzymes were associated with blood ingestion during arthropod hematophagous process, demonstrating their role in avoiding oxidative stress caused by blood intake. Catalase showed to be essential to heme detoxification in Rhipicephalus microplus tick, and diverse antioxidant enzymes are up-regulated after blood intake in a variety of hematophagous parasites. If in one hand ROS is responsible to cell damage and oxidative stress, on the other hand, several works revealed the fundamental role of ROS in cell signaling and function, demonstrating the sophisticated regulation that is necessary to redox homeostasis control.

Conclusions: In the course of arthropods evolution, blood-feeding life style represent a special strategy to acquire energy and biosynthetic precursors. Together with this advantage, hematophagous organisms needed to develop molecular mechanisms to control toxic components ingested with blood. In this context, several works reviewed here identified the role of proteins and enzymes in the control of free heme released during hematophagy and in redox homeostasis, in order to avoid oxidative stress caused by blood intake. This knowledge represents an important contribution in the search for new candidate targets to develop efficient tick control methods.
\end{abstract}

Keywords: tick, redox homeostasis, oxidative stress, hematophagy, reactive oxygen species. 


\section{INTRODUCTION}

\section{THE HEMATOPHAGY PROCESS AND THE CONTROL OF REDOX HOMEOSTASIS IN TICKS AND OTHER HEMATOPHAGOUS}

\section{FREE RADICALS IN REDOX HOMEOSTASIS AND IN THE CONTROL OF OXIDATIVE STRESS}

\section{THE ROLE OF ANTIOXIDANT ENZYMES IN THE REGULATION OF REDOX HOMEOSTASIS}

\section{CONCLUSIONS}

\section{INTRODUCTION}

Ticks are hematophagous ectoparasites that infest a diverse number of vertebrate hosts. The globally distributed Rhipicephalus microplus tick has special importance for veterinary, since it has cattle as preferential host and causes several economical losses in livestock [32]. During blood feeding, this tick stays attached to the host for weeks, causing direct damages to animal health, and indirect damages due to its vector competence in transmit pathogens. Acaricide application is the most commonly used method to control tick in livestock, however, besides causing environmental contamination, the constant use of the same chemical induces the selection of tick resistant populations. Considering these drawbacks, new methods of tick control need to be developed. After recurrent infestations, it was observed that cattle develop some degree of immune resistance against ticks [4]. From this observation, large number of studies demonstrated the use of vaccines as a convenient method against ticks $[19,79,90]$. Although much knowledge has been generated in this scenario, the contribution to understand tick, as well as other arthropods, physiology, genetics and behavior is still necessary to the research progress for vaccines and other alternative methods to tick control.

Among hematophagous arthropods, female ticks have a special ability to blood intake between 50-200 times their initial adult weight [49]. In this way, some works focused on how ticks could handle with this huge amount of ingested blood [15,41,48], since there are toxic components (mainly heme) that can act as an important oxidant agent, damaging biological molecules. Diverse adaptations to blood feeding were described in other hematophagous arthropods, as Rhodnius prolixus [57,58] and Aedes aegypti [66]. Some antioxidant strategies were revealed also in non-hematophagous arthropods, which could help to understand how hematophagous parasites control oxidative damages. In this context, this review intends to highlight the aspects of oxidative molecules and antioxidant systems in biological models and clarify the physiological adaptations used by ticks and others hematophagous parasites to redox homeostasis control, avoiding possible oxidative stress caused by the blood feeding lifestyle.

\section{THE HEMATOPHAGY PROCESS AND THE CONTROL OF REDOX HOMEOSTASIS IN TICKS AND OTHER HEMATOPHAGOUS}

More than 800,000 species of arthropods have been identified and approximately 15,000 have hematophagous habits. It is estimated that hematophagy appeared between 145-65 million years ago in the Jurassic and Cretaceous eras, a strategy that emerged independently at least six times during the arthropods evolutionary course [45]. Blood is a rich source of nutrients: in addition to proteins, it contains sugars, salts, lipids, amino acids and hormones, supplying the arthropod nutritional needs as well as its embryos [35]. Since ticks are able to ingest large amounts of blood, morphological, behavioral, and physiological adaptations of these arthropods in the course of evolution have become essential for the hematophagy success $[31,71,74]$.

Hemoglobin is one of the most abundant proteins in mammalian blood, together with albumin, they comprise about $80 \%$ of blood proteins [82]. During hematophagy, hemoglobin hydrolysis results in the release of large amounts of heme [41], which is the prosthetic group of hemoglobin, as well as heme enzymes [50]. Heme consists of a porphyrin ring formed by an iron $(\mathrm{Fe})$ atom bounded to four nitrogen atoms. $\mathrm{Fe}$ is a transition metal, which may exist in the ferrous $\left(\mathrm{Fe}^{2+}\right)$ or ferric $\left(\mathrm{Fe}^{3+}\right)$ form, and this transient property permits oxidation-reducing catalysis by heme enzymes [70]. On the other hand, heme has a potentially toxic nature: because it is a lipophilic molecule, free heme tends to interact with cell membranes and organelles, destabilizing the cytoskeleton and membrane permeability [76]. Cell lysis was observed after addition of $10 \mathrm{mM}$ of heme to Plasmodium falciparum cell culture [60]. Moreover, due to its reductive nature, free $\mathrm{Fe}$ can easily react with reactive oxygen species (ROS) physiologically produced during metabolic processes. These ROS induce the Fenton reaction, a chain reaction 
that leads to lipid peroxidation and the formation of more ROS [39], which interact with cellular structures damaging proteins [2], lipids [85] and DNA [1]. To avoid such damages, hematophagous arthropods have molecular mechanisms that prevent the triggering of these reactions and the consequent oxidative stress.

A first defense line against heme toxicity in hematophagy consists of physically isolate heme from cellular molecules, reducing its potential reactivity [58]. To do this, during the hemoglobin digestion, released heme molecules are aggregated into organelles that physically separate them from cell molecules. This aggregation mechanism has already been described in several organisms. In P. falciparum it was referred to as malaric pigment [63]. In $R$. prolixus most free heme derived from hemoglobin digestion are accumulated in hemozoins, heme aggregates organized as crystals, which result in the reduction of free radical formation [57,58]. This mechanism was also observed in Schistosoma mansoni helminth and the protozoan Haemoproteus columbae [13]. In the R. microplus tick, where digestion is intracellular and occurs in digestive vesicles, hemoglobin is internalized by receptors endocytosis and, after hemoglobin digestion, the heme is accumulated in organelles called hemosomes, also organized in clusters, but not in the form of crystals [41]. The mosquito Aedes aegypti also aggregates heme into non-crystalline vesicles called hemozoins, and these structures are associated with the peritrophic matrix, a midgut extracellular layer composed of proteins and polysaccharides [66].

Heme-carrying proteins (HCP) is another mechanism that prevents heme to freely react with cell molecules. Diverse arthropods, such as ticks, mosquitoes and hemipters, express HCP $[23,48,59,66]$. Two HCP were well characterized in $R$. prolixus and $R$. microplus named RHBP [20] and HeLp [48], respectively. Although having analogous functions, these proteins have different mechanisms of action, structure and composition, suggesting they evolved independently among these organisms [31]. Besides acting as a defense mechanism against oxidative stress, $\mathrm{HCP}$ are also heme transporters and reservoirs. In $R$. prolixus, RHBP is synthesized in fat body during all stages of development. This protein is secreted into the hemolymph and binds to heme to transport it to the oocytes, where heme plays a key role in embryo development [44]. In R. microplus tick, it has been shown that heme acquisition through diet is essential for the complete embryo development [68]. Heme carrier and storage and transporter proteins are also essential in this process $[42,86]$. Unlike most eukaryotes, ticks lack essential heme biosynthetic enzymes, acquiring all heme by the blood intake [8]. For this reason, heme-carrying proteins play an important role in tick reproduction and development [68]. In vertebrates, hemepoxin, a protein with high heme affinity, play an antioxidant role through the inhibition of heme oxidative reactions $[33,77]$, highlighting the importance of this mechanism even in organisms with low evolutionary relationship.

Hematophagous and non-hematophagous organisms developed adaptations to control free radicals. Non-enzymatic antioxidants are physiologically produced or acquired through the diet. These molecules can act as enzymatic cofactors, as glutathione (GSH), or autonomously, as vitamins. The antioxidant property is due to the ability of donate electrons to oxidized molecules, and so stabilizing reactive species and preventing that these uncontrolled reactions to propagate. Due to electrochemical properties, these antioxidants do not become reactive even after donating electrons. Some arthropods acquired antioxidants through plant sap feeding, which contains tocopherol, carotene and flavonoids, for example [69].

Heme degradation comprises another control mechanism described in hematophagous [83,92]. The heme oxygenase (HO) enzyme catalyzes the heme oxidation into biliverdin, carbon monoxide (CO) and $\mathrm{Fe}^{2+}$, an oxygen and NADPH dependent reaction [40]. In hematophagous, the ferritin enzyme is described as essential for the removal of $\mathrm{Fe}^{2+}$ released from this reaction, avoiding the formation of ROS through Fenton reaction [72]. In contrast, Braz et al. [8] in 1999 , showed that $R$. microplus does not synthesize its own heme and relies solely on the recovery of heme present in the diet. Moreover, it was observed an absence of $\mathrm{HO}$ gene in tick genome, transcriptome and proteome analyses $[17,93,94]$. On the other hand, in D. melanogaster, a non-hematophagous arthropod, heme oxygenase $(\mathrm{HO})$ catabolism also plays a role in tissue formation [18].

Antioxidant enzymes are oxidoreductases with different mechanisms of action, strategically located in specific tissues and cellular microenvironments, responsible to degrade specific reactive species. These enzymes play a key role against ROS and in mainte- 
nance of redox cell homeostasis [80], and since they are found in all animals, they are supposed to play a fundamental role also in non-hematophagous organisms. In R. microplus catalase and GST activities were related with oxygen consumption, lipid peroxidation and oxidative stress during egg and larvae development [28], however, their relationship with hematophagy was also observed. For example, it was demonstrated a role of catalase in the control of hydrogen peroxide (H2O2) levels in $R$. microplus adult females during blood feeding. By inhibiting catalase activity, it was observed an increase in $\mathrm{H}_{2} \mathrm{O}_{2}$ concentration in digestive cell and an impairment in heme detoxification. In vivo, these effects reduced survival and a diminished the oviposition rate [15]. In Ixodes ricinus tick, it has been reported that there are more sulfotransferase transcripts in tick midgut after blood feeding than before [67]. The same pattern was observed with catalase transcripts in Aedes aegypti [56]. In Dermacentor variabilis tick, blood intake promotes the up-regulation of glutathione-S transferase (GST) transcription in midgut and ovary [24]. In A. aegypti, GST transcription increases only after blood feeding, which does not occur after sugar feeding [7]. Moreover, GST was used as a protective vaccine antigen against ticks in diverse works $[55,64,65,73]$, showing its potential to compose an efficient vaccine. In $R$. prolixus, it was described an intense production of $\mathrm{H}_{2} \mathrm{O}_{2}$ and increased expression of superoxide dismutase and catalase enzymes after blood feeding [62]. Inhibition of catalase and synthesis of GSH leads to an increased $\mathrm{H}_{2} \mathrm{O}_{2}$ levels in midgut [62]. Together, these data suggest that antioxidant enzymes act in a network in hematophagous animals, cooperating to control reactive species and preventing increased levels of oxidative stress, which could be caused by large amounts of ingested blood.

\section{FREE RADICALS IN REDOX HOMEOSTASIS AND IN THE CONTROL OF OXIDATIVE STRESS}

Mitochondria is the main source of free radicals in eukaryotic cell, where most of the oxygen $\left(\mathrm{O}_{2}\right)$ is reduced to water. A small part of these $\mathrm{O}_{2}$ molecules is converted into the superoxide anion $\left(\mathrm{O}_{2}\right)$ by the electron transport chain complexes I and III [43]. $\mathrm{O}_{2-}$ results from a single electron reduction of $\mathrm{O}_{2}$ and can damage proteins that contain clusters made up of iron and sulfur metals. High levels of $\mathrm{O}_{2-}$ are more associated with oxidative stress than with cell signaling [75]. It is important to note, however, that $\mathrm{O}_{2-}$ does not damage proteins indiscriminately: there is a specific set of metalloproteins that are sensitive to inactivation by $\mathrm{O}_{2-}$, which then promotes the activation of mechanisms adapted to high levels of $\mathrm{O}_{2-}$ or initiate cell death [14].

The hydroxyl radical $(\mathrm{OH})$ is the most reactive element among all free radicals, since it indiscriminately oxidizes lipids, proteins and DNA, which results in biomolecules damage or genomic instability [21]. This radical is usually generated from $\mathrm{H}_{2} \mathrm{O}_{2}$ in the presence of $\mathrm{Fe}^{+2}$ or $\mathrm{Fe}^{+3}$ ions, triggering the Fenton reaction. Therefore, in addition to free radicals' control, cells also have mechanisms to maintain Fe homeostasis, avoiding the formation of this super reactive radical.

Lipids are the most oxidation susceptible biomolecules, especially the polyunsaturated ones, since the electrons are more exposed, making them vulnerable to free radicals attack. When attacked, phospholipids propagate an oxidative chain reaction in the others plasma membrane phospholipids, promoting the lipid peroxidation propagation and generating different reactive species that are able to attack proteins and DNA. Oxidative damage to these phospholipids, therefore, can lead to cell death not only due to alterations in the plasma membrane, but to functional modifications in proteins and DNA [87]. Malondialdehyde and 4-hydroxynonenal are products of lipid peroxidation and may cause DNA mutations [46] and functional protein alterations $[6,10]$, respectively. These protein oxidative modifications can affect JNK and caspase-3 kinases, activating the mechanisms of cell death induced by lipid peroxidation $[6,10]$.

Proteins are attacked by reactive species in a reversible or irreversible way. Although virtually all amino acids can react with free radicals, lysine, arginine, histidine, proline, threonine and cysteine are especially susceptible due to their biochemical properties [87]. High oxidative stress can induce disulfide bonds formation between cysteines, or secondary oxidative modifications such as oxidized proteins conjugation (protein adducts), which may lead to the accumulation of protein complexes that inactivate proteasomes, leading to cell death [37].

In comparison to proteins and lipids, DNA is less susceptible to oxidative modifications due to its compartmentalized double-helix structure, histone proteins and other DNA-bound proteins that protect its nucleophilic centers. DNA susceptibility to damage occurs in highly electronegative $\mathrm{O}$ and $\mathrm{N}$ atoms from purines and pyrimidines. In addition, its double bonds 
are the primary targets of $\mathrm{OH}$ radical. Reactions are mainly directed to $\mathrm{C} 5$ and $\mathrm{C} 6$ in pyrimidines and $\mathrm{C} 4$ and $\mathrm{C} 8$ in purines. The oxidative damage caused by subtraction of $\mathrm{H}$ causes a rupture in the double strand [9]. On the other hand, mitochondrial DNA is more susceptible to oxidative damage, since it is closer to the ROS produced in mitochondria and does not have so effective repair mechanisms as the nuclear DNA [36].

Although highly reactive, free radicals have some substrate specificity, as previously mentioned. In addition, compartmentalization of ROS production in the cell is an important determinant of cell signaling or oxidative stress production [38]. Proteins that act on redox signaling pathways need to be closer to radicals' production. For example, the targets of $\mathrm{H}_{2} \mathrm{O}_{2}$ generated from plasma membrane NADPH oxidases are also located on the plasma membrane, allowing these signaling pathways to be activated, and the same can be observed in mitochondria [3].

\section{THE ROLE OF ANTIOXIDANT ENZYMES IN THE REGULATION OF REDOX HOMEOSTASIS}

Although it was first observed that free radicals cause cell damage, it has been reviewed that, in moderate concentrations, reactive species are involved in physiological responses, such as cell signaling and immune response $[47,75]$. In fact, a new perspective shows that an anti/pro-oxidant balance is required for cellular homeostasis, and a number of mechanisms evolved to regulate reactive species [25]. In this sense, antioxidant enzymes act as one of the main reduction systems in the organisms, controlling oxidative damages and regulating cell signaling.

Damages caused by free radicals are consequence of its chemical nature. Due to a higher electronegativity, oxygen and nitrogen strongly attract electrons from nearby molecules. Since molecules with unpaired electrons are reactive species, they tend to sequester electrons from other compounds in order to become stable, triggering oxidative chain reactions. Antioxidants are molecules that act by donating electrons necessary to stabilize these reactive species, and then the antioxidant itself becomes a reactive specie. The antioxidant enzymes act through oxidationreduction (redox) reactions, reducing the reactive species through the oxidation of another compound and keeping this compound in a non-reactive form.

Catalase is an enzyme involved in ROS regulation, it is a tetrameric heme peroxidase (degrades $\mathrm{H}_{2} \mathrm{O}_{2}$ ) mostly present in peroxisomes [29], which are multifunctional organelles involved in oxidation of various biomolecules and are essential to fatty acid $\beta$-oxidation long chain, purine catabolism and biosynthesis of glycerolipids, steroids and bile acids [27]. Because these metabolic activities are a powerful ROS source, the enzymatic antioxidant activity in this organelle has a central role. Catalases directly dismutate two $\mathrm{H}_{2} \mathrm{O}_{2}$ on two $\mathrm{H}_{2} \mathrm{O}$ and one $\mathrm{O}_{2}$ molecules. This activity depends essentially on three components: the active site heme portion, a reduced NADPH bound to the NADPHbinding domain and a structure formed by long peptide loops that interact when it is in tetrameric form [95]. Its catalytic activity occurs in a two-stage mechanism dependent on the versatile iron states, e.g. ferrous $\left(\mathrm{Fe}^{+2}\right)$, ferric $\left(\mathrm{Fe}^{+3}\right)$, compound $\mathrm{I}\left(\mathrm{Fe}^{4+} \mathrm{O}\right)$, compound II $\left(\mathrm{Fe}^{4+}-\mathrm{OH}\right)$ and compound III $\left(\mathrm{Fe}^{3+}-\mathrm{OOH}\right)$. In the first stage, the $\mathrm{Fe}^{+3}$ bounded to the heme group acts as a reducing agent, reducing a molecule of $\mathrm{H}_{2} \mathrm{O}_{2}$ into water, generating $\mathrm{Fe}^{+4}$, which bounds to an oxygen atom. This intermediate product is called compound $\mathrm{I}$. In the second stage, another $\mathrm{H}_{2} \mathrm{O}_{2}$ molecule is used as a reducing agent to regenerate the $\mathrm{Fe}^{+3}$, using $\mathrm{Fe}^{+4}$ from the catalytic center, also producing another molecule of $\mathrm{H}_{2} \mathrm{O}$ and $\mathrm{O}_{2}$ [95]. NADPH possibly has a protection role during catalase activity, preventing the formation of enzyme inactivated forms [30].

Superoxide dismutase (SOD) is an antioxidant metalloenzyme that dismutes $\mathrm{O}_{2-}$ (superoxide) to the less reactive products $\mathrm{O}_{2}$ and $\mathrm{H}_{2} \mathrm{O}_{2}$. Superoxide is the product of some signaling enzymes, as well as the byproduct of various metabolic processes, including mitochondrial respiration. All aerobic organisms present SOD located in different cellular and subcellular locations, reflecting the multiple $\mathrm{O}_{2-}$ sources in the cell. Four classes of SOD have evolved in these organisms and possess different catalytic metal ions: $\mathrm{Cu} / \mathrm{Zn}-\mathrm{SOD}$, Mn-SOD, Fe-SOD and, the most recently discovered in bacteria, Ni-SOD [51]. Eukaryotic organisms' express $\mathrm{Cu} / \mathrm{Zn}-\mathrm{SOD}$ in the cytoplasm and secrete it into the extracellular medium. Mn/Fe-SODs is typically present in mitochondria, one of the largest sources of $\mathrm{O}_{2-}$ in the cell [91]. The evolutionary reason for the existence of SODs coupled with different metal ions could be related to the different availability of these metal compounds in the biosphere during different geological eras [11]. They all perform a similar mechanism, in which an $\mathrm{O}_{2-}$ molecule acts reducing the transition metal to form $\mathrm{O}_{2}$, and then another $\mathrm{O}_{2}$ oxidizes the transition 
metal to generate $\mathrm{H}_{2} \mathrm{O}_{2}$. In this reaction, a source of hydrogen from an adjacent $\mathrm{H}_{2} \mathrm{O}$ molecule is required for $\mathrm{O}_{2}$ reduction into $\mathrm{H}_{2} \mathrm{O}_{2}[11]$.

Peroxidases comprise a group of enzymes that reduce $\mathrm{H}_{2} \mathrm{O}_{2}$ based on different catalytic mechanisms. Two essential peroxidases that work on redox homeostasis are glutathione and thioredoxin peroxidases. These enzymes use, respectively, GSH and thioredoxin ( $\operatorname{Trx}$ ) as cofactors for reducing power sources. Electrons are transferred from these cofactors to the reactive species through peroxidase catalysis, and then are restored by reductase enzymes that use electrons from NADPH. In this mechanism, enzymes act in a coupled form (peroxidasereductase), maintaining an active system capable of self-recycling [26]. Glutathione peroxidase (GPx) has an active selenocysteine residue at its catalytic center, which can reduce both $\mathrm{H}_{2} \mathrm{O}_{2}$ in $\mathrm{H}_{2} \mathrm{O}$ and $\mathrm{O}_{2}$, and lipid hydroperoxides $(\mathrm{LOOH})$ in $\mathrm{H}_{2} \mathrm{O}_{2}$ and lipid alcohols ( $\mathrm{LOH}$ ), using two GSH molecules as electron donors. Oxidized glutathione (GSSG) is formed in this reaction, which can be regenerated to GSH through the activity of glutathione reductase or exported to the extracellular medium [34]. GPx acts in different compartments: cytosol, nucleus [54] and mitochondria [89], which suggest its wide cellular distribution. It has a special function related to the reduction of $\mathrm{LOOH}[12,88]$, due to their conformation and monomeric structure, which confer easy access to larger hydroperoxides that could not be catalyzed by tetrameric peroxidases. In this sense, it plays a fundamental role in the maintenance of membrane integrity, avoiding lipid peroxidation $[16,78]$.

Transferases are another class of enzymes that act in the elimination of xenobiotics, drugs and endogenous components. GST and sulfotransferases act in the conjugation of GSH [5] and sulfate [22], respectively, to toxic compounds. Therefore, they have a virtual infinite variety of substrates, facilitating their excretion after this modification. Although most studies correlate GSTs activity with drug resistance in several organisms [52], more recent works still reveal a nonclassical GST activity, such as peroxidase $[61,81,96]$. The sulfotransferases are also related to the inactivation of hormones in mammals [53,84], a mechanism commonly found in other eukaryotes.

\section{CONCLUSIONS}

The data presented in this review highlight that the understanding of how hematophagous arthropods maintain redox cellular homeostasis can help in the development of parasites' control methods. Several mechanisms were developed along with evolution to adapt the blood feeding habit and, despite the higher amounts of ingested blood and its potentially toxicity, hematophagous parasites can handle it, maintaining the physiological balance between ROS production and elimination and avoid oxidative stress. Despite antioxidant enzymes have a certain substrate specificity, their numerous isoforms present in hematophagous organisms suggest that they can act on several substrates, possibly located in various subcellular compartments, which demonstrates the sophisticated ROS control methods by these organisms. Moreover, these enzymes are part of fundamental cellular processes, not only related with ROS detoxification and redox homeostasis, but also with cell signaling, acting as compensatory or complementary systems within biological systems.

Acknowledgments. This work was supported by grants from CNPq-Instituto Nacional de Ciência e Tecnologia de Entomologia Molecular, CAPES, CNPq, FAPERJ and FAPERGS.

Declaration of interest. The authors report no conflicts of interest. The authors alone are responsible for the content and writing of this manuscript.

\section{REFERENCES}

1 Aft R.L. \& Mueller G.C. 1983. Hemin-mediated DNA strand scission. The Journal of Biological Chemistry. 258(19): 12069-12072.

2 Aft R.L. \& Mueller G.C. 1984. Hemin-mediated oxidative degradation of proteins. The Journal of Biological Chemistry. 259(1): 301-305.

3 Al-Mehdi A.B., Pastukh V.M., Swiger B.M., Reed D.J., Patel M.R., Bardwell G.C., Pastukh V.V., Alexeyev M.F. \& Gillespie M.N. 2012. Perinuclear mitochondrial clustering creates an oxidant-rich nuclear domain required for hypoxia-induced transcription. Science Signaling. 5(231): 47-67. 
4 Allen J.R. 1994. Host resistance to ectoparasites. Revue Scientifique et Technique. 13(4): 1287-1303.

5 Angelucci F., Baiocco P., Brunori M., Gourlay L., Morea V. \& Bellelli A. 2005. Insights into the catalytic mechanism of glutathione S-transferase: the lesson from Schistosoma haematobium. Structure. 13(9): 1241-1246.

6 Awasthi Y.C., Sharma R., Cheng J.Z., Yang Y., Sharma A., Singhal S.S. \& Awasthi S. 2003. Role of 4-hydroxynonenal in stress-mediated apoptosis signaling. Molecular Aspects of Medicine. 24(4-5): 219-230.

7 Bottino-Rojas V., Talyuli O.A., Jupatanakul N., Sim S., Dimopoulos G., Venancio T.M., Bahia A.C., Sorgine M.H., Oliveira P.L. \& Paiva-Silva G.O. 2015. Heme Signaling Impacts Global Gene Expression, Immunity and Dengue Virus Infectivity in Aedes aegypti. PLoS One. 10(8): e0135985.

8 Braz G.R., Coelho H.S., Masuda H. \& Oliveira P.L. 1999. A missing metabolic pathway in the cattle tick Boophilus microplus. Current Biology. 9(13): 703-706.

9 Breen A.P. \& Murphy J.A. 1995. Reactions of oxyl radicals with DNA. Free Radical Biology \& Medicine. 18(6): 1033-1077.

10 Breitzig M., Bhimineni C., Lockey R. \& Kolliputi N. 2016. 4-Hydroxy-2-nonenal: a critical target in oxidative stress? American Journal of Physiology. Cell physiology. 311(4): 537-543.

11 Case A.J. 2017. On the Origin of Superoxide Dismutase: An Evolutionary Perspective of Superoxide-Mediated Redox Signaling. Antioxidants. 6(4): 82.

12 Cavas L. \& Tarhan L. 2003. Glutathione redox system, GSH-Px activity and lipid peroxidation (LPO) levels in tadpoles of R.r.ridibunda and B.viridis. Cell Biochemistry and Function. 21(1): 75-79.

13 Chen M.M., Shi L. \& Sullivan Jr. D.J. 2001. Haemoproteus and Schistosoma synthesize heme polymers similar to Plasmodium hemozoin and beta-hematin. Molecular and Biochemical Parasitology. 113(1): 1-8.

14 Chen Y., Azad M.B. \& Gibson S.B. 2009. Superoxide is the major reactive oxygen species regulating autophagy. Cell Death and Differentiation. 16(7): 1040-1052.

15 Citelli M., Lara F.A., da Silva Vaz Jr. I. \& Oliveira P.L. 2007. Oxidative stress impairs heme detoxification in the midgut of the cattle tick, Rhipicephalus (Boophilus) microplus. Molecular and Biochemical Parasitology. 151(1): 8188.

16 Cozza G., Rossetto M., Bosello-Travain V., Maiorino M., Roveri A., Toppo S., Zaccarin M., Zennaro L. \& Ursini F. 2017. Glutathione peroxidase 4-catalyzed reduction of lipid hydroperoxides in membranes: The polar head of membrane phospholipids binds the enzyme and addresses the fatty acid hydroperoxide group toward the redox center. Free Radical Biology \& Medicine. 112: 1-11.

17 Cramaro W.J., Revets D., Hunewald O.E., Sinner R., Reye A.L. \& Muller C.P. 2015. Integration of Ixodes ricinus genome sequencing with transcriptome and proteome annotation of the naive midgut. BMC Genomics. 16: 871.

18 Cui L., Yoshioka Y., Suyari O., Kohno Y., Zhang X., Adachi Y., Ikehara S., Yoshida T., Yamaguchi M. \& Taketani S. 2008. Relevant expression of Drosophila heme oxygenase is necessary for the normal development of insect tissues. Biochemical and Biophysical Research Communications. 377(4): 1156-1161.

19 da Silva Vaz Jr. I., Logullo C., Sorgine M., Velloso F.F., Rosa de Lima M.F., Gonzales J.C., Masuda H., Oliveira P.L. \& Masuda A. 1998. Immunization of bovines with an aspartic proteinase precursor isolated from Boophilus microplus eggs. Veterinary Immunology and Immunopathology. 66(3-4): 331-341.

20 Dansa-Petretski M., Ribeiro J.M., Atella G.C., Masuda H. \& Oliveira P.L. 1995. Antioxidant role of Rhodnius prolixus heme-binding protein. Protection against heme-induced lipid peroxidation. The Journal of Biological Chemistry. 270(18): 10893-10896.

21 Dizdaroglu M. \& Jaruga P. 2012. Mechanisms of free radical-induced damage to DNA. Free Radical Research. 46(4): 382-419.

22 Dong D., Ako R. \& Wu B. 2012. Crystal structures of human sulfotransferases: insights into the mechanisms of action and substrate selectivity. Expert Opinion on Drug Metabolism \& Toxicology. 8(6): 635-646.

23 Donohue K.V., Khalil S.M., Sonenshine D.E. \& Roe R.M. 2009. Heme-binding storage proteins in the Chelicerata. Journal of Insect Physiology. 55(4): 287-296.

24 Dreher-Lesnick S.M., Mulenga A., Simser J.A. \& Azad A.F. 2006. Differential expression of two glutathione Stransferases identified from the American dog tick, Dermacentor variabilis. Insect Molecular Biology. 15(4): 445-453.

25 Espinosa-Diez C., Miguel V., Mennerich D., Kietzmann T., Sanchez-Perez P., Cadenas S. \& Lamas S. 2015. Antioxidant responses and cellular adjustments to oxidative stress. Redox Biology. 6: 183-197. 
26 Flohe L. \& Ursini F. 2008. Peroxidase: a term of many meanings. Antioxid Redox Signal. 10(9): 1485-1490.

27 Fransen M., Lismont C. \& Walton P. 2017. The Peroxisome-Mitochondria Connection: How and Why? International Journal of Molecular Sciences. 18(6):1126-1150.

28 Freitas D.R., Rosa R.M., Moraes J., Campos E., Logullo C., Da Silva Vaz Jr. I. \& Masuda A. 2007. Relationship between glutathione S-transferase, catalase, oxygen consumption, lipid peroxidation and oxidative stress in eggs and larvae of Boophilus microplus (Acarina: Ixodidae). Comparative Biochemistry and Physiology. Part A, Molecular \& Integrative Physiology. 146(4): 688-694.

29 Gasselhuber B., Carpena X., Graf M.M., Pirker K.F., Nicolussi A., Sundermann A., Hofbauer S., Zamocky M., Furtmuller P.G., Jakopitsch C., Oostenbrink C., Fita I. \& Obinger C. 2015. Eukaryotic Catalase-Peroxidase: The Role of the Trp-Tyr-Met Adduct in Protein Stability, Substrate Accessibility, and Catalysis of Hydrogen Peroxide Dismutation. Biochemistry. 54(35): 5425-5438.

30 Goyal M.M. \& Basak A. 2010. Human catalase: looking for complete identity. Protein Cell. 1(10): 888-897.

31 Graca-Souza A.V., Maya-Monteiro C., Paiva-Silva G.O., Braz G.R., Paes M.C., Sorgine M.H., Oliveira M.F. \& Oliveira P.L. 2006. Adaptations against heme toxicity in blood-feeding arthropods. Insect Biochemistry and Molecular Biology. 36(4): 322-335.

32 Grisi L., Leite R.C., Martins J.R., Barros A.T., Andreotti R., Cancado P.H., Leon A.A., Pereira J.B. \& Villela H.S. 2014. Reassessment of the potential economic impact of cattle parasites in Brazil. Revista Brasileira de Parasitologia Veterinaria. 23(2): 150-156.

33 Gutteridge J.M. \& Smith A. 1988. Antioxidant protection by haemopexin of haem-stimulated lipid peroxidation. Biochemical Journal. 256(3): 861-865.

34 Hirrlinger J., Konig J., Keppler D., Lindenau J., Schulz J.B. \& Dringen R. 2001. The multidrug resistance protein MRP1 mediates the release of glutathione disulfide from rat astrocytes during oxidative stress. Journal of Neurochemistry. 76(2): 627-636.

35 Hocking B. 1971. Blood-sucking behavior of terrestrial arthropods. Annual Review of Entomology. 16: 1-26.

36 Inoue M., Sato E.F., Nishikawa M., Park A.M., Kira Y., Imada I. \& Utsumi K. 2003. Mitochondrial generation of reactive oxygen species and its role in aerobic life. Current Medicinal Chemistry. 10(23): 2495-2505.

37 Jung T., Hohn A. \& Grune T. 2014. The proteasome and the degradation of oxidized proteins: Part II - protein oxidation and proteasomal degradation. Redox Biology. 2: 99-104.

38 Kaludercic N., Deshwal S. \& Di Lisa F. 2014. Reactive oxygen species and redox compartmentalization. Frontiers in Physiology. 5: 285.

39 Kehrer J.P. 2000. The Haber-Weiss reaction and mechanisms of toxicity. Toxicology. 149(1): 43-50.

40 Kikuchi G., Yoshida T. \& Noguchi M. 2005. Heme oxygenase and heme degradation. Biochemical and Biophysical Research Communications. 338(1): 558-567.

41 Lara F.A., Lins U., Paiva-Silva G., Almeida I.C., Braga C.M., Miguens F.C., Oliveira P.L. \& Dansa-Petretski M. 2003. A new intracellular pathway of haem detoxification in the midgut of the cattle tick Boophilus microplus: aggregation inside a specialized organelle, the hemosome. Journal of Experimental Biology. 206(Pt 10): 1707-1715.

42 Lara F.A., Pohl P.C., Gandara A.C., Ferreira J. da S., Nascimento-Silva M.C., Bechara G.H., Sorgine M.H., Almeida I.C., Vaz Jr. I. da S. \& Oliveira P.L. 2015. ATP Binding Cassette Transporter Mediates Both Heme and Pesticide Detoxification in Tick Midgut Cells. PLoS One. 10(8): e0134779.

43 Le Bras M., Clement M.V., Pervaiz S. \& Brenner C. 2005. Reactive oxygen species and the mitochondrial signaling pathway of cell death. Histology and Histopathology. 20(1): 205-219.

44 Machado E.A., Oliveira P.L., Moreira M.F., de Souza W. \& Masuda H. 1998. Uptake of Rhodnius heme-binding protein (RHBP) by the ovary of Rhodnius prolixus. Arch Insect Biochem Physiol. 39(4): 133-143.

45 Mans B.J., Louw A.I. \& Neitz A.W. 2002. Evolution of hematophagy in ticks: common origins for blood coagulation and platelet aggregation inhibitors from soft ticks of the genus Ornithodoros. Molecular Biology and Evolution. 19(10): 1695-1705.

46 Marnett L.J. 1999. Lipid peroxidation-DNA damage by malondialdehyde. Mutation Research. 424(1-2): 83-95.

47 Martin K.R. \& Barrett J.C. 2002. Reactive oxygen species as double-edged swords in cellular processes: low-dose cell signaling versus high-dose toxicity. Human \& Experimental Toxicology. 21(2): 71-75. 
48 Maya-Monteiro C.M., Daffre S., Logullo C., Lara F.A., Alves E.W., Capurro M.L., Zingali R., Almeida I.C. \& Oliveira P.L. 2000. HeLp, a heme lipoprotein from the hemolymph of the cattle tick, Boophilus microplus. The Journal of Biological Chemistry. 275(47): 36584-36589.

49 Mehlhorn H. 2016. Encyclopedia of Parasitology. 3rd edn. New York: Springer, 3084p.

50 Mense S.M. \& Zhang L. 2006. Heme: a versatile signaling molecule controlling the activities of diverse regulators ranging from transcription factors to MAP kinases. Cell Research. 16(8): 681-692.

51 Miller A.F. 2012. Superoxide dismutases: ancient enzymes and new insights. FEBS Letters. 586(5): 585-595.

52 Morrow C.S. \& Cowan K.H. 1990. Glutathione S-transferases and drug resistance. Cancer Cells. 2(1): 15-22.

53 Mueller J.W., Gilligan L.C., Idkowiak J., Arlt W. \& Foster P.A. 2015. The Regulation of Steroid Action by Sulfation and Desulfation. Endocrine Reviews. 36(5): 526-563.

54 Muse K.E., Oberley T.D., Sempf J.M. \& Oberley L.W. 1994. Immunolocalization of antioxidant enzymes in adult hamster kidney. The Histochemical journal. 26(9): 734-753.

55 Ndawula Jr. C., Sabadin G.A., Parizi L.F. \& da Silva Vaz Jr. I. 2019. Constituting a glutathione S-transferase-cocktail vaccine against tick infestation. Vaccine. 37(14): 1918-1927.

56 Oliveira J.H.M., Talyuli O.A.C., Gonçalves R.L.S., Paiva-Silva G.O., Sorgine M.H.F., Alvarenga P.H. \& Oliveira P.L. 2017. Catalase protects Aedes aegypti from oxidative stress and increases midgut infection prevalence of Dengue but not Zika. PLOS Neglected Tropical Diseases. 11(4): 1-13.

57 Oliveira M.F., Silva J.R., Dansa-Petretski M., de Souza W., Lins U., Braga C.M., Masuda H. \& Oliveira P.L. 1999. Haem detoxification by an insect. Nature. 400(6744): 517-518.

58 Oliveira M.F., Timm B.L., Machado E.A., Miranda K., Attias M., Silva J.R., Dansa-Petretski M., de Oliveira M.A., de Souza W., Pinhal N.M., Sousa J.J., Vugman N.V. \& Oliveira P.L. 2002. On the pro-oxidant effects of haemozoin. FEBS Letters. 512(1-3): 139-144.

59 Oliveira P.L., Kawooya J.K., Ribeiro J.M., Meyer T., Poorman R., Alves E.W., Walker F.A., Machado E.A., Nussenzveig R.H., Padovan G.J. \& Masuda H. 1995. A heme-binding protein from hemolymph and oocytes of the blood-sucking insect, Rhodnius prolixus. Isolation and characterization. The Journal of Biological Chemistry. 270(18): 10897-10901.

60 Orjih A.U., Banyal H.S., Chevli R. \& Fitch C.D. 1981. Hemin lyses malaria parasites. Science 214(4521): 667-669.

61 Ortelli F., Rossiter L.C., Vontas J., Ranson H. \& Hemingway J. 2003. Heterologous expression of four glutathione transferase genes genetically linked to a major insecticide-resistance locus from the malaria vector Anopheles gambiae. The Biochemical Journal. 373(Pt 3): 957-963.

62 Paes M.C., Oliveira M.B. \& Oliveira P.L. 2001. Hydrogen peroxide detoxification in the midgut of the blood-sucking insect, Rhodnius prolixus. Archives of Insect Biochemistry and Physiology. 48(2): 63-71.

63 Pagola S., Stephens P.W., Bohle D.S., Kosar A.D. \& Madsen S.K. 2000. The structure of malaria pigment betahaematin. Nature. 404(6775): 307-310.

64 Parizi L.F., Reck Jr. J. , Oldiges D.P., Guizzo M.G., Seixas A., Logullo C., de Oliveira P.L., Termignoni C., Martins J.R. \& Vaz Jr. I.S. 2012. Multi-antigenic vaccine against the cattle tick Rhipicephalus (Boophilus) microplus: a field evaluation. Vaccine. 30(48): 6912-6917.

65 Parizi L.F., Utiumi K.U., Imamura S., Onuma M., Ohashi K., Masuda A. \& da Silva Vaz Jr. I. 2011. Cross immunity with Haemaphysalis longicornis glutathione S-transferase reduces an experimental Rhipicephalus (Boophilus) microplus infestation. Experimental Parasitology. 127(1): 113-118.

66 Pascoa V., Oliveira P.L., Dansa-Petretski M., Silva J.R., Alvarenga P.H., Jacobs-Lorena M. \& Lemos F.J. 2002. Aedes aegypti peritrophic matrix and its interaction with heme during blood digestion. Insect Biochemistry and Molecular Biology. 32(5): 517-523.

67 Perner J., Provaznik J., Schrenkova J., Urbanova V., Ribeiro J.M. \& Kopacek P. 2016. RNA-seq analyses of the midgut from blood- and serum-fed Ixodes ricinus ticks. Scientific Reports. 6: 36695.

68 Perner J., Sobotka R., Sima R., Konvickova J., Sojka D., Oliveira P.L., Hajdusek O. \& Kopacek P. 2016. Acquisition of exogenous haem is essential for tick reproduction. Elife. 5: e12318.

69 Pisoschi A.M., Pop A., Cimpeanu C. \& Predoi G. 2016. Antioxidant Capacity Determination in Plants and PlantDerived Products: A Review. Oxidative Medicine and Cellular Longevity. 2016: 1-13.

70 Poulos T.L. 2014. Heme enzyme structure and function. Chemical Reviews. 114(7): 3919-3962. 
71 Ribeiro J.M. 1987. Role of saliva in blood-feeding by arthropods. Annual Review of Entomology. 32: 463-478.

72 Ryter S.W. \& Tyrrell R.M. 2000. The heme synthesis and degradation pathways: role in oxidant sensitivity. Heme oxygenase has both pro- and antioxidant properties. Free Radical Biology \& Medicine. 28(2): 289-309.

73 Sabadin G.A., Parizi L.F., Kiio I., Xavier M.A., da Silva Matos R., Camargo-Mathias M.I., Githaka N.W., Nene V. \& da Silva Vaz Jr. I. 2017. Effect of recombinant glutathione S-transferase as vaccine antigen against Rhipicephalus appendiculatus and Rhipicephalus sanguineus infestation. Vaccine. 35(48 Pt B): 6649-6656.

74 Sanders H.R., Evans A.M., Ross L.S. \& Gill S.S. 2003. Blood meal induces global changes in midgut gene expression in the disease vector, Aedes aegypti. Insect Biochemistry and Molecular Biology. 33(11): 1105-1122.

75 Schieber M. \& Chandel N.S. 2014. ROS function in redox signaling and oxidative stress. Current Biology. 24(10): 453-462.

76 Schmitt T.H., Frezzatti Jr. W.A. \& Schreier S. 1993. Hemin-induced lipid membrane disorder and increased permeability: a molecular model for the mechanism of cell lysis. Archives of Biochemistry and Biophysics. 307(1): 96-103.

77 Seery V.L. \& Muller-Eberhard U. 1973. Binding of porphyrins to rabbit hemopexin and albumin. The Journal of Biological Chemistry. 248(11): 3796-3800.

78 Seiler A., Schneider M., Forster H., Roth S., Wirth E.K., Culmsee C., Plesnila N., Kremmer E., Radmark O., Wurst W., Bornkamm G.W., Schweizer U. \& Conrad M. 2008. Glutathione peroxidase 4 senses and translates oxidative stress into 12/15-lipoxygenase dependent- and AIF-mediated cell death. Cell Metabolism. 8(3): 237-248.

79 Seixas A., Oliveira P., Termignoni C., Logullo C., Masuda A. \& da Silva Vaz Jr. I. 2012. Rhipicephalus (Boophilus) microplus embryo proteins as target for tick vaccine. Veterinary Immunology and Immunopathology. 148(1-2): 149-156.

80 Sen C.K. 2001. Antioxidant and redox regulation of cellular signaling: introduction. Medicine \& Science in Sports \& Exercise. 33(3): 368-370.

81 Singh S.P., Coronella J.A., Benes H., Cochrane B.J. \& Zimniak P. 2001. Catalytic function of Drosophila melanogaster glutathione S-transferase DmGSTS1-1 (GST-2) in conjugation of lipid peroxidation end products. European Journal of Biochemistry. 268(10): 2912-2923.

82 Sojka D., Pytelkova J., Perner J., Horn M., Konvickova J., Schrenkova J., Mares M. \& Kopacek P. 2016. Multienzyme degradation of host serum albumin in ticks. Ticks Ticks and Tick-borne Diseases. 7(4): 604-613.

83 Spencer C.S., Yunta C., de Lima G.P.G., Hemmings K., Lian L.Y., Lycett G. \& Paine M.J.I. 2018. Characterisation of Anopheles gambiae heme oxygenase and metalloporphyrin feeding suggests a potential role in reproduction. Insect Biochemistry and Molecular Biology. 98: 25-33.

84 Suzuki-Anekoji M., Suzuki A., Wu S.W., Angata K., Murai K.K., Sugihara K., Akama T.O., Khoo K.H., Nakayama J., Fukuda M.N. \& Fukuda M. 2013. In vivo regulation of steroid hormones by the Chst10 sulfotransferase in mouse. The Journal of Biological Chemistry. 288(7): 5007-5016.

85 Tappel A.L. 1955. Unsaturated lipide oxidation catalyzed by hematin compounds. The Journal of Biological Chemistry. 217(2): 721-733.

86 Thompson D.M., Khalil S.M., Jeffers L.A., Sonenshine D.E., Mitchell R.D., Osgood C.J. \& Michael Roe R. 2007. Sequence and the developmental and tissue-specific regulation of the first complete vitellogenin messenger RNA from ticks responsible for heme sequestration. Insect Biochemistry and Molecular Biology. 37(4): 363-374.

87 Trachootham D., Lu W., Ogasawara M.A., Nilsa R.D. \& Huang P. 2008. Redox regulation of cell survival. Antioxid Redox Signal. 10(8): 1343-1374.

88 Ursini F., Maiorino M., Valente M., Ferri L. \& Gregolin C. 1982. Purification from pig liver of a protein which protects liposomes and biomembranes from peroxidative degradation and exhibits glutathione peroxidase activity on phosphatidylcholine hydroperoxides. Biochimica et Biophysica Acta. 710(2): 197-211.

89 Utsunomiya H., Komatsu N., Yoshimura S., Tsutsumi Y. \& Watanabe K. 1991. Exact ultrastructural localization of glutathione peroxidase in normal rat hepatocytes: advantages of microwave fixation. The journal of histochemistry and cytochemistry : official journal of the Histochemistry Society. 39(9): 1167-1174.

90 Vaz Junior I.S., Martinez R.H., Oliveira A., Heck A., Logullo C., Gonzales J.C., Dewes H. \& Masuda A. 1996. Functional bovine immunoglobulins in Boophilus microplus hemolymph. Veterinary Parasitology. 62(1-2): 155-160.

91 Weisiger R.A. \& Fridovich I. 1973. Superoxide dismutase. Organelle specificity. The Journal of biological chemistry. 248(10): 3582-3592. 
92 Wigglesworth V.B. 1943. The Fate of Haemoglobin in Rhodnius prolixus (Hemiptera) and other Blood-Sucking Arthropods. Proceedings of the Royal Society. 131 (6): 313-339

93 Xavier M.A., Tirloni L., Pinto A.F.M., Diedrich J.K., Yates 3rd J.R., Gonzales S., Farber M., da Silva Vaz Jr. I. \& Termignoni C. 2019. Tick Gene's organ engagement in lipid metabolism revealed by a combined transcriptomic and proteomic approach. Ticks and Tick Borne Diseases. 10(4): 787-797.

94 Xavier M.A., Tirloni L., Pinto A.F.M., Diedrich J.K., Yates 3rd J.R., Mulenga A., Logullo C., da Silva Vaz Jr. I., Seixas A. \& Termignoni C. 2018. A proteomic insight into vitellogenesis during tick ovary maturation. Scientific Reports. 8(1): 4698-4712.

95 Zamocky M., Furtmuller P.G. \& Obinger C. 2008. Evolution of catalases from bacteria to humans. Antioxidants \& redox signaling. 10(9): 1527-1548.

96 Ziegler R. \& Ibrahim M.M. 2001. Formation of lipid reserves in fat body and eggs of the yellow fever mosquito, Aedes aegypti. Journal of Insect Physiology. 47(6): 623-627. 